\title{
The fate of volatiles during magma body crystallization
}

\section{SARA VULPIUS AND LENA NOACK}

\section{Freie Universität Berlin}

Presenting Author: sara.vulpius@fu-berlin.de

After the solidification of the magma ocean, outgassing from the interior is the prevailing volatile source of early Earth's atmosphere. Besides the well-studied extrusive degassing, this process also includes the often neglected intrusive volatile release. Although, intrusive magma production rates are assumed to be significantly higher compared to extrusive rates. This renders the investigation and quantification of possible volatile exsolution mechanisms crucial.

The process of fractional crystallization within a magma body has an influence on the solubility and thus on the associated volatile release. Due to cooling of an intrusion, nominally anhydrous minerals precipitate from the melt. These minerals mainly incorporate elements that are compatible with their crystal lattice. Since volatiles such as $\mathrm{H}_{2} \mathrm{O}$ and $\mathrm{CO}_{2}$ behave like incompatible elements, they accumulate in the remaining melt. At a certain point, the melt is saturated and the exsolution of a volatile phase initiates. The solubility of the volatiles is determined by several parameters like the lithostatic and the partial pressure, the temperature and the melt composition. In this study, we investigate the effect of these parameters as well as the impact of the volatile accumulation due to fractional crystallization on the solubility and the related volatile exsolution. We focus on the release of $\mathrm{H}_{2} \mathrm{O}$ and $\mathrm{CO}_{2}$ from basaltic magma bodies within the lithosphere. Figure 1 displays the proportion of $\mathrm{H}_{2} \mathrm{O}$ (blue) and $\mathrm{CO}_{2}$ (red) dissolved in the melt as a function of pressure. Considering fractional crystallization (right), the solubility of $\mathrm{H}_{2} \mathrm{O}$ at 1000 bar is reduced by about 60 $\%$ and that of $\mathrm{CO}_{2}$ by even $\sim 90 \%$. The left figure is a reproduction from Gaillard and Scaillet 2014 [1]. To determine the fate of the accumulating volatiles, we compare the density of the developing melt with the density of the host rock. If the host rock has a higher density, the liquid phase (melt and volatiles) will ascent either directly to the surface or to shallower levels of the crust. Furthermore, we take into account the possible effect of the precipitation of hydrous minerals (e.g., amphibole) during the crystallization process.

[1] Gaillard and Scaillet (2014) EPSL 403, 307-316.
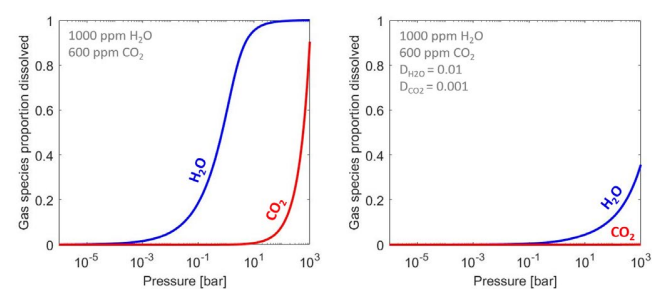